Revista de Metalurgia

Enero-Marzo 2018, 54 (1), e114

ISSN-L: 0034-8570

https://doi.org/10.3989/revmetalm.114

\title{
Caracterización microestructural y modelización mediante elementos finitos de uniones soldadas de la aleación de Magnesio AZ31
}

\author{
José A. Segarra ${ }^{\mathrm{a}, \bigotimes}$, Antonio Portolés ${ }^{\mathrm{a}}$ \\ ${ }^{a}$ E.T.S. de Ingenieros Industriales, Departamento de Física Aplicada e Ingeniería de Materiales, \\ Universidad Politécnica de Madrid, Calle de José Gutiérrez Abascal, 2, 28006 Madrid \\ ${ }^{\square}$ Autor para la correspondencia: jsegarra79@gmail.com
}

Enviado: 25 Enero 2016; Aceptado: 24 Noviembre 2017; Publicado on-Line: 19 Marzo 2018

\begin{abstract}
RESUMEN: En este artículo se ha investigado de qué manera la microestructura de las aleaciones de magnesio AZ31 se ve afectada por los ciclos térmicos que se producen durante los procesos de soldadura, tratando de modelizar mediante programas de elementos finitos los ciclos térmicos que se producen en estos materiales. Las muestras estudiadas han sido soldadas mediante soldadura TIG (Tungsten Inert Gas) y con diferentes materiales de aporte. En el estudio se ha empleado microscopía óptica para analizar la microestructura, microscopía electrónica de barrido y software de simulación. El estudio realizado indica por un lado que en este tipo de aleaciones los microconstituyentes más comunes son compuestos Al-Mn o Al-Mn-Mg, no observándose la presencia de la fase beta $\left(\mathrm{Mg}_{17} \mathrm{Al}_{12}\right)$ a temperatura ambiente, por otro lado los modelos de simulación que se han obtenido indican que para las zonas que alcanzan temperaturas máximas del orden de $550{ }^{\circ} \mathrm{C}$ se produce la cristalización del material base y también sería el límite de las zona de disolución de los precipitados de composición Al-Mn o Al-Mn-Mg, que parecen actuar como inhibidores de la corrosión.
\end{abstract}

PALABRAS CLAVE: AZ31; Corrosión; Elementos finitos; Microestructura; Microscopía electrónica; Precipitados; SEM; TIG

Citar como/Citation: Segarra, J.A.; Portolés, A. (2018). "Caracterización microestructural y modelización mediante elementos finitos de uniones soldadas de la aleación de Magnesio AZ31”. Rev. Metal. 54(1): e114. https://doi.org/10.3989/ revmetalm.114

ABSTRACT: Microstructural characterization and finite element modeling of AZ31 magnesium alloys welded joints. In this article, it has been studied how the microstructure of AZ31 magnesium alloy can be affected by the thermic cycles produced by welding processes, trying to modeling by element finite software the thermic cycles in this material. The AZ31 samples tested were welded using Gas Tugsten Arc Welding (GTAW) and different filler materials. For this investigation, optic microscopy, scanning electronic microscopy, and finite elements method software has been used. This work indicates in one hand that in this type of alloys the microconstituyentes are Al-Mn o Al-Mn-Mg compounds, the presence of $\beta$-phase cannot be found at room temperature in this research at room, on the other hand the obtained simulation models indicate that the recrystallization takes place in the areas which reach maximum temperatures around $550{ }^{\circ} \mathrm{C}$, this value is also the limit of the dissolution area for the Al-Mn o $\mathrm{Al}-\mathrm{Mn}-\mathrm{Mg}$ precipitated particles which are very likely to act as inhibitors of the corrosion in $\mathrm{NaCl}$ electrolytes.

KEYWORDS: AZ31; Corrosion; Finite element method; GTAW; MEF; Microstructure; Precipitated compounds; Scanning electronic microscopy

ORCID: José A. Segarra (https://orcid.org/0000-0003-3513-0297); Antonio Portolés (https://orcid.org/0000-00031224-0821)

Copyright: (C) 2018 CSIC. Este es un artículo de acceso abierto distribuido bajo los términos de la licencia de uso y distribución Creative Commons Reconocimiento 4.0 Internacional (CC BY 4.0). 


\section{INTRODUCCÍON}

El magnesio y sus aleaciones constituyen un grupo de materiales muy interesante desde el punto de vista de la ingeniería. Su ligereza, su resistencia a la fatiga, estabilidad dimensional, su resistencia alcalina son propiedades con múltiples aplicaciones, pero su mal comportamiento frente a la corrosión dificulta su empleo, (Avedesiam y Baker, 1999).

Otro de los aspectos que hacen interesante este grupo de materiales es su disponibilidad, las reservas mundiales de magnesio son muy importantes, de acuerdo con Kramer (2001), sólo las reservas mundiales de magnesita $\left(\mathrm{MgCO}_{3}\right)$ se estiman en unas 3400 millones de toneladas, mientras que las reservas obtenibles a partir de agua marina o depósitos salinos son difíciles de calcular.

Pero el magnesio y sus aleaciones es un grupo de materiales que puede experimentar corrosión por diferentes procesos (corrosión galvánica, corrosión por picaduras, filiforme, corrosión bajo tensiones, corrosión fatiga...) tal y como resumen Zeng et al. (2006), y su conformabilidad también presenta problemas.

Quizá los inconvenientes indicados en el párrafo anterior sean la explicación de porqué en la actualidad, y pese a que el magnesio sigue siendo un metal importante desde el punto de metalúrgico o químico cuya producción aumentan cada año, se está produciendo un reducción en el número de aplicaciones individuales, es decir, productos enteramente fabricados en Mg o sus aleaciones (Polmear, 2006).

En este artículo, en línea con el anteriormente publicado por estos autores (Segarra et al., 2015), se ha investigado, tratando de justificar mediante programas de simulación, de qué manera puede verse afectada la estructura de las uniones soldadas de aleación AZ31B y como los ciclos térmicos inducidos durante este proceso modifican la microestructura de estos materiales.

El empleo de modelos de simulación y el desarrollo de modelos matemáticos como el desarrollado por Te-Chang et al. (2011) para el estudio del efecto de los tratamientos térmicos en este tipo de aleaciones, son herramientas que pueden ayudar al investigador o al diseñador a entender mejor el comportamiento de los materiales evitando problemas, siempre teniendo en cuenta que este tipo de herramientas van a darnos una visión precisa, uniforme y homogénea de una realidad que es imprecisa, discontinua y heterogénea .

Otro hecho destacable deducido del estudio de las diferentes referencias bibliográficas publicadas recientemente, es la disparidad de resultados de las caracterizaciones microestructurales a la hora de definir el tipo o naturaleza de estos compuestos precipitados presentes en la aleación AZ31; mientras algunos autores indican que estos serían partículas que fase $\beta \mathrm{Mg}_{17} \mathrm{Al}_{12}$, otros hablan de compuestos Al-Mn, compuestos ternarios Al-Mn-Mg incluso Mg-Si. En la Tabla 1 se incluye el resume los compuestos precipitados identificados en la aleación AZ31 ordenados por referencias bibliográficas.

Por todos estos motivos se decidió combinar técnicas experimentales como la microscopía óptica, microscopía electrónica de barrido y microdureza con modelos de simulación basados en métodos de elementos finitos, de modo que se puedan identificar que fases y microconstituyentes aparecen en este tipo de uniones y proponer hipótesis que expliquen su comportamiento en respuesta al ciclo térmico producido por la soldadura.

Los objetivos de este estudio consistieron en caracterizar la microestructura de la aleación AZ31B una vez soldada, identificando las diferentes fases y microconstituyentes mediante microscopía óptica y electrónica, desarrollar un modelo de simulación térmico que permitiera explicar el comportamiento de la soldadura de estas aleaciones y que desde el punto de vista del diseño y la durabilidad permita tomar decisiones de cara a la optimización de los diseños.

\section{PROCEDIMIENTOS EXPERIMENTALES}

\subsection{Materiales}

Para este estudio se emplearon probetas soldadas de aleación AZ31B H-24, endurecida por deformación y parcialmente recocida, que fueron soldadas con diferentes materiales de aporte. Las probetas soldadas eran chapas de dimensiones 200x150x3,2 $\mathrm{mm}$, estas dimensiones fueron las empleadas en los modelos de simulación.

Como materiales de aporte se emplearon varilla de aleación de magnesio tipo AZ31B y AZ92 de 4 mm de diámetro, Avedesiam y Baker (1999), recogen en su trabajo que para la soldadura de aleaciones del sistema $\mathrm{Mg}-\mathrm{Al}$ es recomendable el empleo de

TABLA 1. Compuestos identificados en muestras de aleación AZ31 según diferentes autores

\begin{tabular}{|c|c|c|c|c|}
\hline \multicolumn{5}{|c|}{ Compuestos identificados } \\
\hline$\beta \mathrm{Mg}_{17} \mathrm{Al}_{12}$ & $\mathrm{AlMn}_{2}$ & $\mathrm{Al}_{8} \mathrm{Mn}_{5}$ & $\left.\operatorname{Mg}_{2} \operatorname{Si~Mg} g_{32}(\mathrm{Al}, \mathrm{Zn})_{49}\right]$ & $\mathrm{Al}_{19} \mathrm{Mn}_{4}, \mathrm{Al}_{11} \mathbf{M n}_{4}$ \\
\hline Dobrzanski et al. (2007) & Pardo et al. (2008) & Ben Hamu et al. (2009) & Ben Hamu et al. (2009) & Merino et al. (2010) \\
\hline Cheng et al. (2009) & Feliu et al. (2011) & Merino et al. (2010) & & \\
\hline Walton et al. (2012) & Merino et al. (2010) & Song y Xu (2012) & & \\
\hline
\end{tabular}


materiales de aporte con un contenido de aluminio mayor que el del material base para reducir la sensibilidad de las uniones soldadas frente a la fisuración en caliente, aunque esta recomendación es lógica desde un punto de vista metalúrgico dado el aluminio mejora la resistencia mecánica, la dureza y amplía el rango de fusión del material fundido, el efecto de la composición disimilar del material de aporte en el comportamiento frente a la corrosión parece no ser tan beneficioso, tal y como indican otros estudios (Segarra et al., 2015). Por este motivo en anteriores publicaciones se decidió incluir como materiales de aporte el mismo que el del material base y el más disimilar recomendado en la referencia previa.

Las muestras se soldaron con sistema TIG con corriente alterna con una tensión de $17 \mathrm{~V}$ y con una intensidad de $120 \mathrm{~A}$, la velocidad soldadura fue de $3 \cdot 10^{-3} \mathrm{~m} \cdot \mathrm{s}^{-1}$.

Para este estudio se emplearon probetas de cortes transversales de las zonas soldadas incluyendo el cordón, la zona afectada térmicamente y material base.

\subsection{Microscopia óptica}

El estudio micrográfico se realizó con microscopia óptica con un banco metalográfico Nikon Eclipse L150, provisto de objetivos Nikon Lu Plan de 5, 10, 50 y 100 aumentos, el tratamiento de imágenes se realizó con el software Perfect Image v7.7.

Para determinar las dimensiones de la zona afectada térmicamente se emplearon diferentes micrografías, previamente tratadas con el programa Perfect Image v7.7, una vez escaladas correctamente, fueron empleadas como referencias externas con el programa.

Para la medición del tamaño de grano se empleó el software GRANI. V1.1.0. de Clara Vision que permite determinar el tamaño de grano a partir de la norma ISO-643 (UNE-EN ISO 643, 2013).

Para determinar las dimensiones del cordón de cara a la modelización del proceso de soldadura se empleó un medidor de perfiles NIKON V-12 .

\subsection{Microscopia electrónica de barrido}

La microscopía electrónica se realizó mediante un microscopio electrónico de barrido JEOL JSM 6400 , de una resolución de imagen de $3,5 \mathrm{~nm}(25 \mathrm{kV}$ a $8 \mathrm{~mm}$ de distancia de trabajo) provisto de detector de electrones retrodispersados (resolución de la imagen, $10 \mathrm{~nm}$ a $8 \mathrm{~mm}$ ) y sonda para análisis EDS (resolución de $133 \mathrm{eV}$ para análisis elemental cualitativo).

Las muestras estudiadas fueron preparadas superficialmente, lijadas y pulidas con alúmina, la preparación previa de las probetas para la microscopía de barrido se realizó con un recubrimiento por evaporación de grafito y posterior metalización con oro.
Se realizaron observaciones de las zonas más representativas del cordón, zona afectada térmicamente y material base, analizando químicamente las diferentes fases y microconstituyentes observados.

\subsection{Microdureza}

Para la caracterización mecánica de los materiales se optó por el estudio de la microdureza, ya que permite caracterizar con más detalle las propiedades mecánicas de las diferentes zonas presentes en la soldadura.

La microdureza se determinó realizando varias líneas de medidas a lo largo de la zona analizada (MB, ZAT o Cordón). Estos ensayos se realizaron con un microdurómetro LECO M-400 con una carga de $100 \mathrm{~g}$.

\subsection{Modelización mediante elementos finitos}

Para la modelización y simulación del proceso de soldadura se decidió el empleo de un del software comercial y basado en el método de elementos finitos de probada eficacia y fiabilidad.

El mallado o discretización de la geometría se realizó partiendo de las dimensiones reales de las muestras soldadas que fueron trasladadas a un modelo definido mediante coordenadas, posteriormente fueron importadas al programa de simulación (Fig. 1), dentro del modelo mallado, se definieron los diferentes grupos de elementos junto con las propiedades físicas y mecánicas en función temperatura junto con los parámetros referidos a la conductividad térmica la convención y la radiación implicados en el problema térmico, el modelo general se desarrolló considerando la variable temporal y la temperatura, de forma que las propiedades físicas y los inputs térmicos de cada grupo de elementos fueran variando en función del tiempo y de la temperatura.

Como condiciones de contorno se emplearon la temperatura ambiental, y las restricciones al movimiento impuestas por las fijaciones durante la soldadura de las muestras o elementos auxiliares como las chapas de respaldo.

Los modelos se fueron ajustando hasta obtener una buena convergencia con las medidas reales (microscopía, proyección de perfiles) de las diferentes zonas de la soldadura, material base, cordón y zona afectada térmicamente como puede apreciarse en la Fig. 2.

\section{RESULTADOS}

Con los resultados de los modelos de simulación y los resultados obtenidos de la microscopia óptima y electrónica de barrido se llegó a las siguientes conclusiones: 


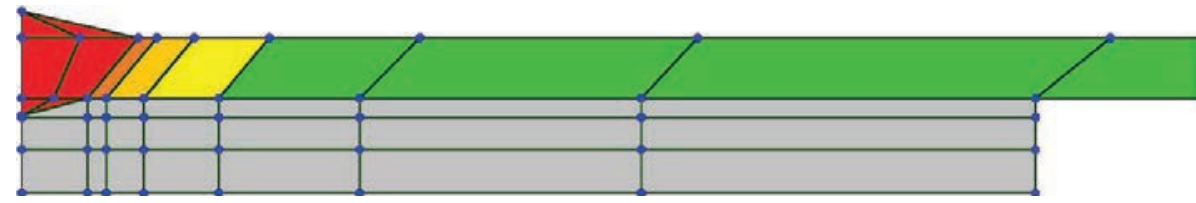

Figura 1. Malla de elementos y nodos (en azul) empleado en la modelización de las uniones soldadas.
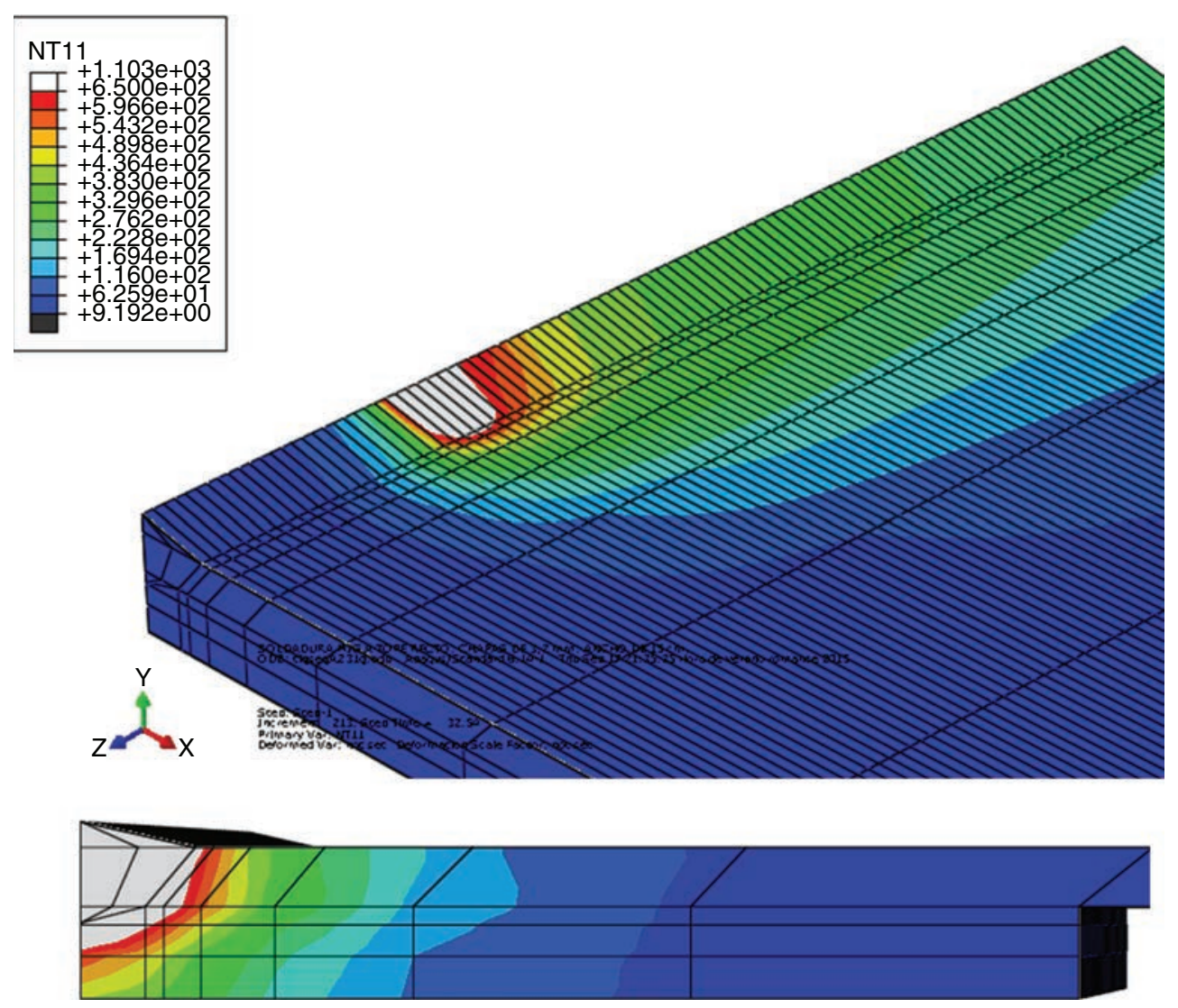

FIGURA 2. Resultados del modelo de simulación. Vista general y sección transversal.

\subsection{Modelos de simulación y ciclos térmicos}

Los modelos de simulación desarrollados muestran las diferencias en los ciclos y gradientes térmicos que experimentan cada uno de los elementos del modelo de la simulación.

Se observan diferencias claras entre los modelos en los que se utiliza como material de aporte la aleación AZ31 y la aleación AZ92, debido a que la temperatura de fusión de esta primera es del orden de los $630{ }^{\circ} \mathrm{C}$ mientras que la de la segunda es del orden de $595^{\circ} \mathrm{C}$, al fundirse parte del material base, la experiencia indica que la temperatura de fusión real se encuentra en valor promedio, $610-615^{\circ} \mathrm{C}$, el baño de fusión de las primeras es menor que el de las segundas, tal y como puede observarse en la Fig. 3, según los modelos de simulación la franja de material sometido a alta temperatura sin llegar al estado de fusión es menor en el caso de las soldaduras en las que se emplea aleación AZ92 confirmando los observado en los ensayos de microscopia óptica, de igual forma se aprecia que dicha franja de material que se correspondería con la zona afectada térmicamente permanece más tiempo alta temperatura, las diferencias son pequeñas en valor absoluto, pero sustanciales si se comparan con la duración total del ciclo térmico que experimenta la zona afectada térmicamente.

En la Fig. 4, se muestran los ciclos térmicos para dos muestras soldadas con material de aporte diferente, indicándose estas diferencias; en ordenadas la franja comprendida entre la temperatura de fusión 

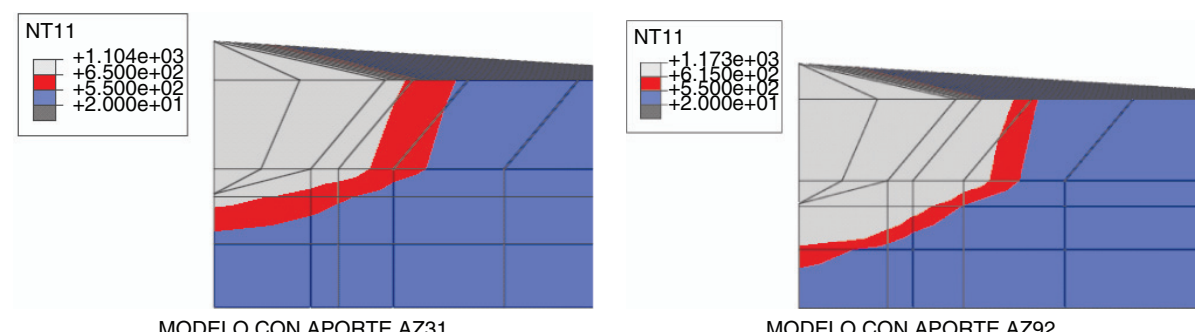

Figura 3. Comparativa del tamaño del cordón de acuerdo con los modelos de simulación en función del material deporte empleado.
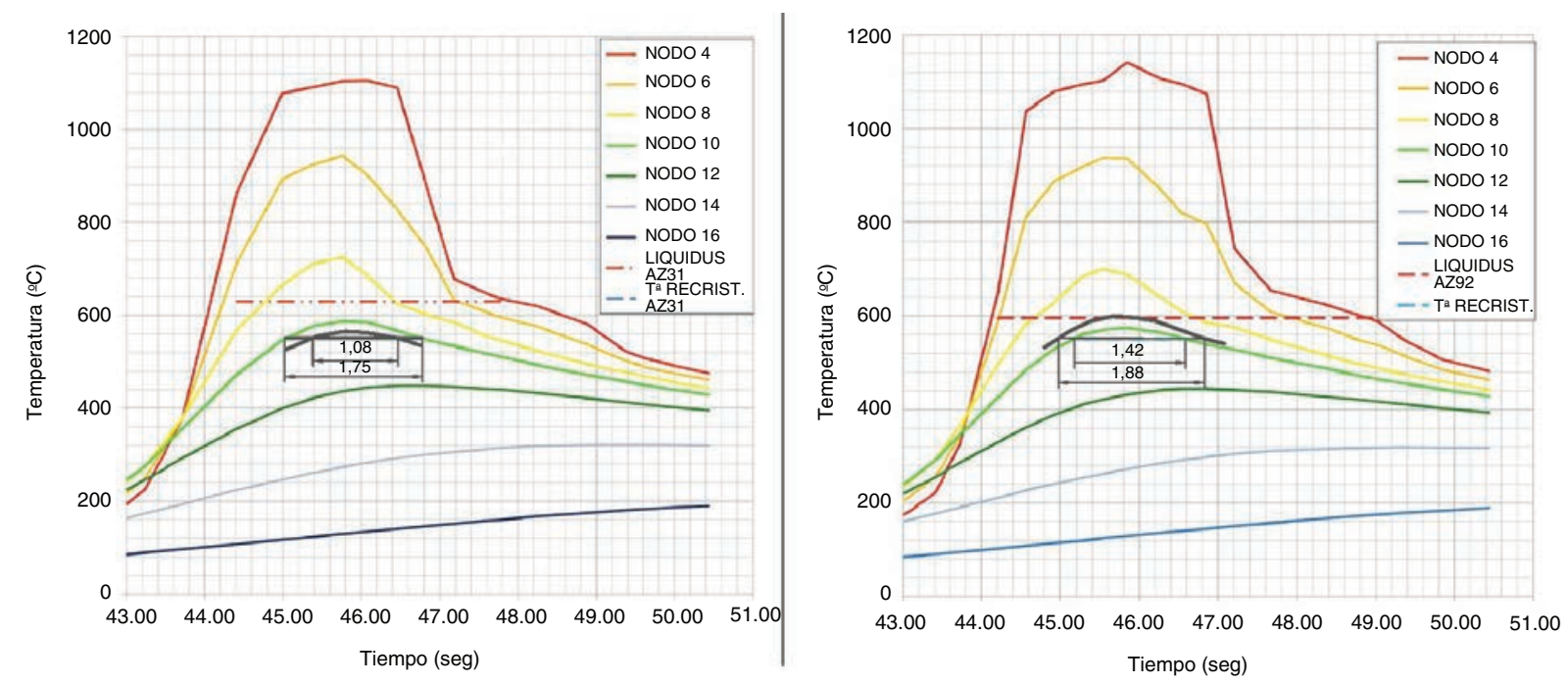

FIGURA 4. Comparativa de los ciclos térmicos empleando como material de aporta aleación AZ31 y AZ92 para los nodos de la cara superior.

y la temperatura de recristalización, temperatura que posteriormente se justificará con los resultados de la observación microscópica de las muestras, da una idea de las dimensiones del cordón de soldadura, mientras que en caso de las abscisas, se puede determinar durante cuánto tiempo el material base permanece a temperaturas superiores a las de recristalización, sin llegar alcanzar la temperatura de fusión.

Con este modelo térmico y con las soluciones que proporciona se desarrolló un modelo mecánico en el que las diferentes zonas de la soldadura se representan a través de los cambios en las propiedades físicas del material en función de la temperatura y del tiempo.

\subsection{Caracterización de la microestructura. Microscopia electrónica de barrido}

Las muestras fueron observadas mediante microscopia óptica para determinar las dimensiones de la zona afectada térmicamente, como se puede apreciar en la Fig. 5, las muestras soldadas con material de aporte AZ31 presentan zonas afectadas térmicamente de mayores dimensiones que las soldadas con material de aporte AZ92, comparando los resultados obtenidos en la simulación con los de la microscopia óptica se infiere que para la aleación AZ31B la zona afectada térmicamente se produce para aquellos elementos que experimentaron ciclos térmicos cuya temperatura máxima sobrepasa los $550^{\circ}$ siempre que no se supere la temperatura de líquidus.

Partiendo de los resultados de los ensayos de microscopía óptica se realizaron observaciones con microscopia electrónica de barrido y análisis químicos a efectos de determinar la composición química de las diferentes fases precipitadas en diferentes zonas de las probetas de las soldaduras (Fig. 6).

Los resultados del análisis elemental cualitativo indican tal y como se recoge en la Tabla 2 que las partículas precipitadas tanto en la zona del material base como en la zona del cordón de las muestras soldadas son compuestos Al-Mn, 


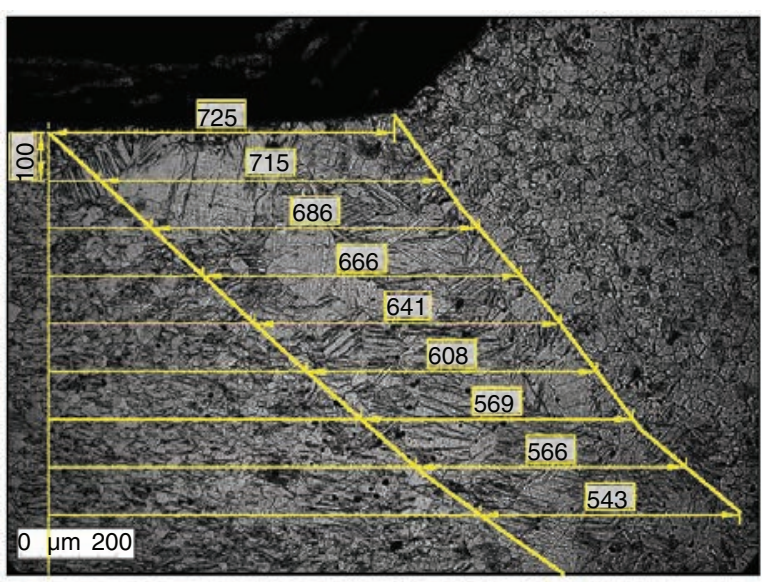

APORTE AZ31

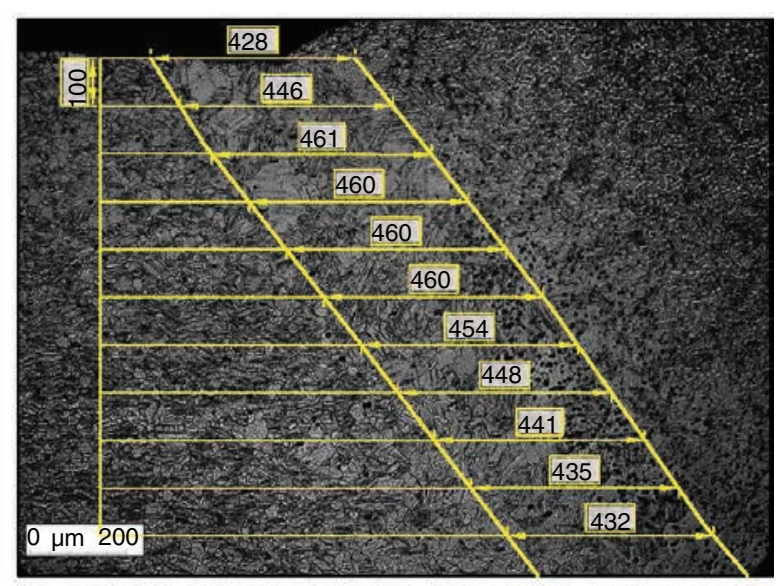

APORTE AZ92

FIGURA 5. Comparativa de las dimensiones de las ZAT para muestras soldadas con diferente material de aporte.

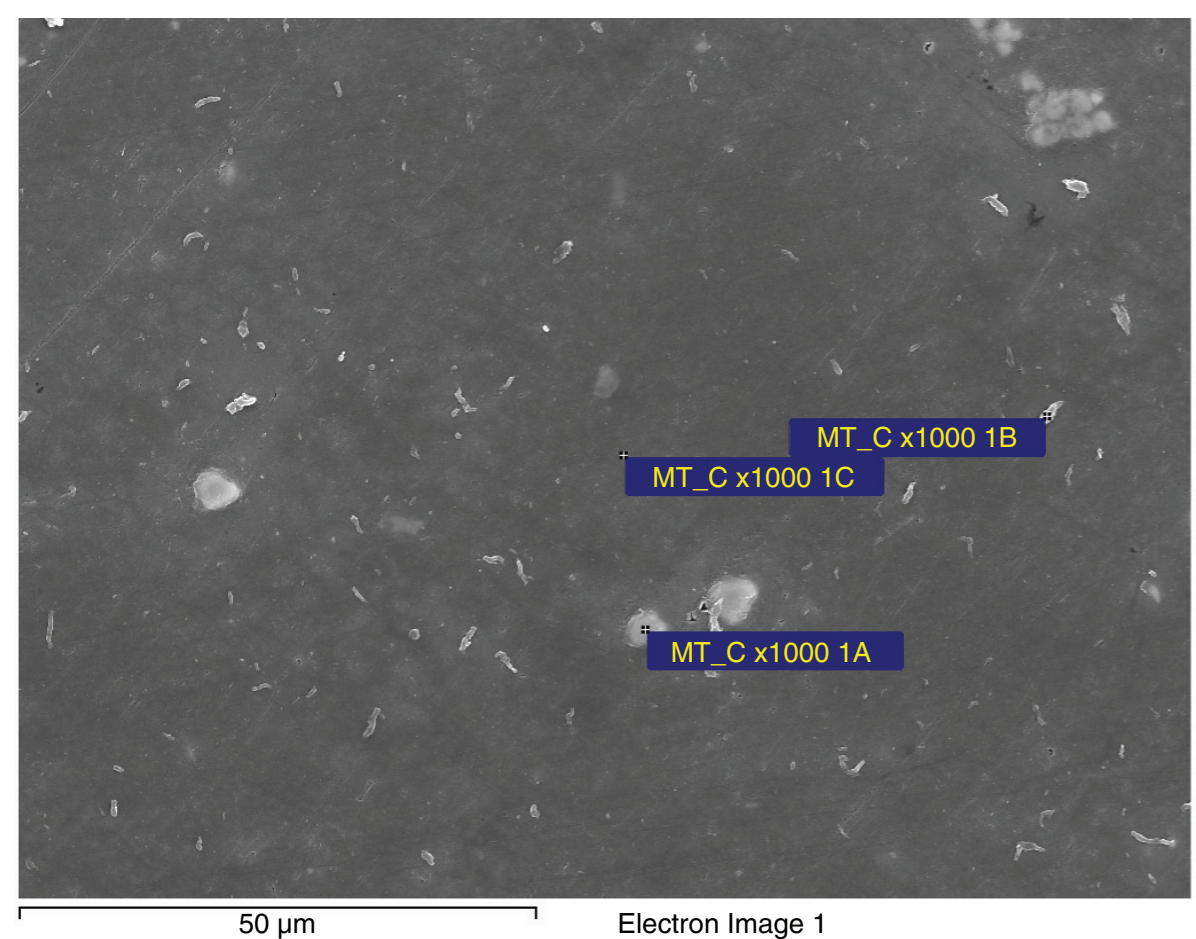

FIgURA 6. Imagen SEM de la zona del cordón de soldadura (1000 aumentos), fases y microconstituyentes identificados mediante microscopía electrónica. Zona del cordón.

o Al-Mn-Mg, sin que se haya podido identificar la fase $\beta \mathrm{Mg}_{17} \mathrm{Al}_{12}$. De igual forma, y en línea con lo publicado anteriormente por estos autores (Segarra et al., 2015), se ha observado que en la zona afectada térmicamente la cantidad de precipitados es menor (Fig. 7a) que en el caso de la zona del cordón y del material base

\subsection{Ensayos mecánicos}

Los ensayos mecánicos muestran que las variaciones de las propiedades mecánicas en función del material de aporte empleado no son muy grandes. Tal y como se puede observar en la Fig. 8 la microdureza promedio del material base ronda los $60 \mathrm{HV}$ 
TABLA 2. Estequiometria de las diferentes partículas precipitadas identificadas mediante espectroscopia de difracción de rayos $\mathrm{X}$ en función del material de aporte empleado la soldadura y de la zona de esta

\begin{tabular}{lccc}
\hline \multirow{2}{*}{$\begin{array}{l}\text { Material } \\
\text { de aporte }\end{array}$} & \multicolumn{3}{c}{ Zona de la soldadura } \\
\cline { 2 - 4 } & Cordón & ZAT & Material base \\
\hline $\mathrm{AZ92}$ & $\mathrm{MgO}, \mathrm{Mg}_{2} \mathrm{Al}_{3} \mathrm{Mn}_{2}$ & - & $\mathrm{MnAl}_{2}, \mathrm{Mg}_{7} \mathrm{Al}_{3} \mathrm{Mn}$ \\
$\mathrm{AZ31}$ & $\mathrm{Mg}_{2} \mathrm{Al}_{3} \mathrm{Mn}_{2}$ & - & $\mathrm{MnAl}_{2}, \mathrm{Mg}_{7} \mathrm{Al}_{3} \mathrm{Mn}$ \\
\hline
\end{tabular}

mientras que en el caso del cordón y la zona afectada térmicamente está más cercana a los $50 \mathrm{HV}$. Los valores se mueven en el rango esperable para una aleación en estado H24, endurecida por deformación y parcialmente recocida, según se indica en el trabajo de Avedesiam y Baker (1999), aunque no se observa mejora aparente de las propiedades mecánicas en la zona del cordón de soldadura en las muestras soldadas con AZ92. (a)

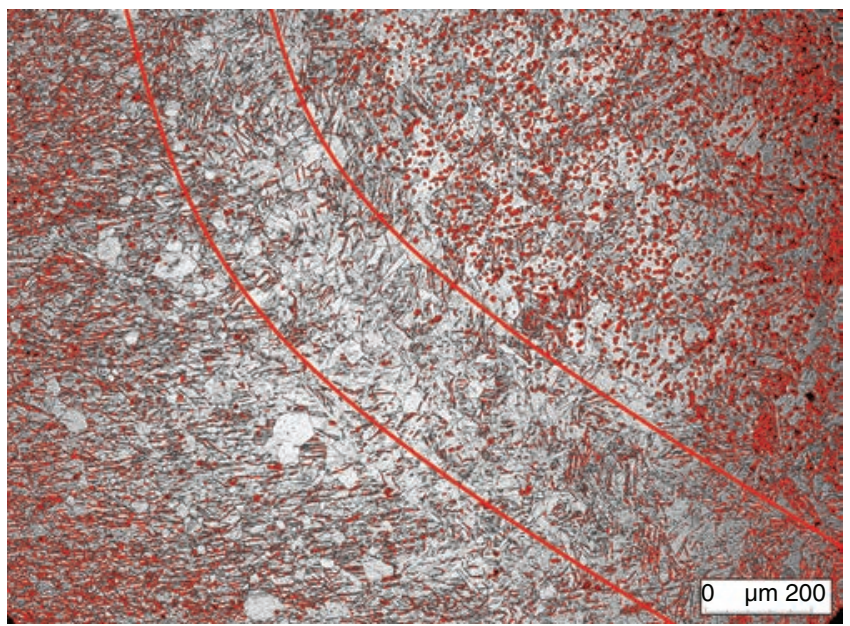

(b)

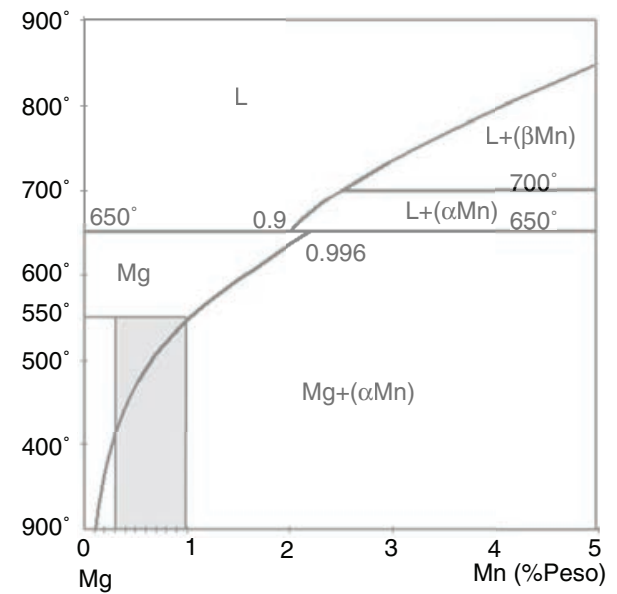

FiguRA 7. a) Micrografía 100 aumentos de detalle donde se indican en rojo la diferente proporción de partículas dispersas entre material base/cordón y la ZAT; b) Detalle de diagrama binario Mg-Mn indicando la variación de solubilidad del Mn en la franja de composición de la AZ31B y el hipotético térmico de solubilización (valores del diagrama binario según Avedesiam y Baker (1999) citando a otros autores).

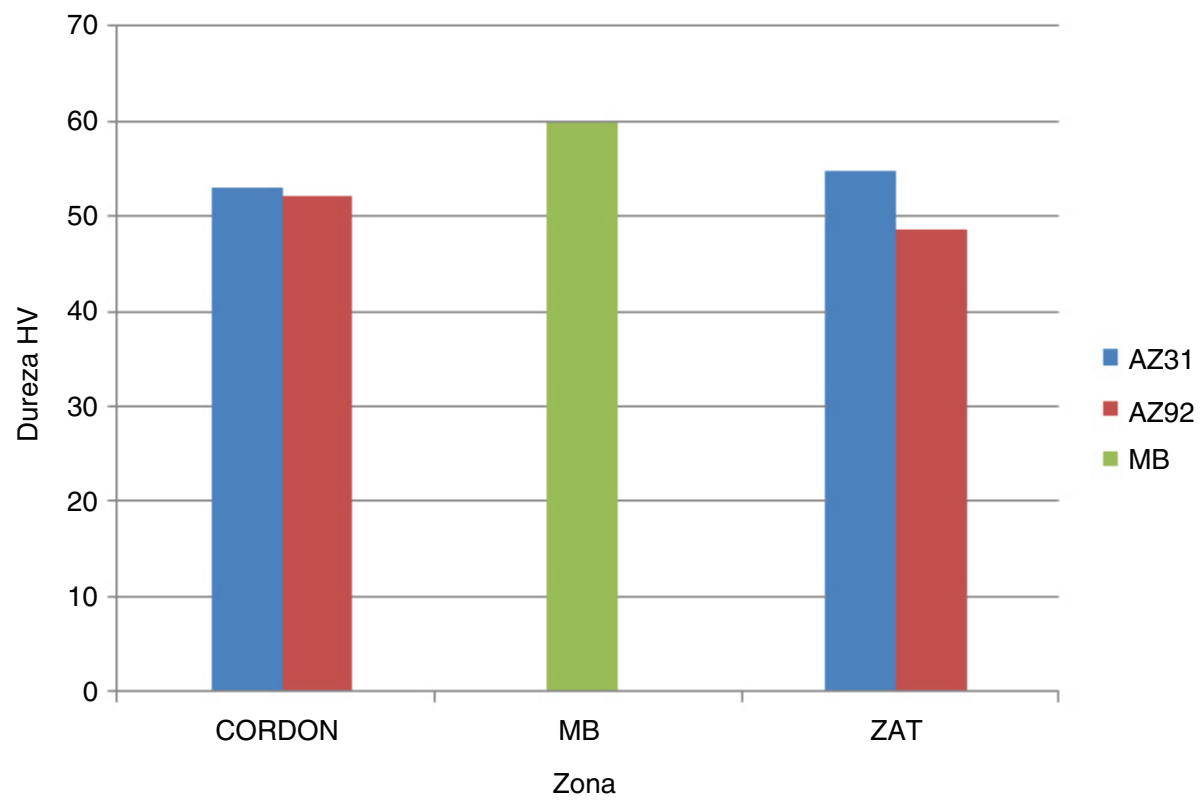

FIGURA 8. Valores promedio de dureza Vickers para diferentes zonas en función de material de aporte empleado. 


\section{DISCUSIÓN DE RESULTADOS}

El modelo termomecánico desarrollado para esta investigación permite deducir en las condiciones de soldeo descritas en el apartado de materiales cuál va a ser la microestructura de la soldadura con bastante fiabilidad.

El modelo permite explicar las diferencias el tamaño de grano observadas en la zona afectada térmicamente en anteriores investigaciones y justificar porque las zonas afectadas térmicamente de las muestras soldadas con material de aporte AZ31 son de menor de mayor tamaño que las que emplean como material de aporte aleación AZ92, de igual manera se puede justificar la diferencia del tamaño de grano ya que de acuerdo a las simulaciones los ciclos térmicos en el caso de las muestras soldadas con aleación AZ92 indican que el material permanece alta temperatura durante más tiempo favoreciendo el crecimiento de grano.

Las observaciones mediante microscopía electrónica de barrido confirman la ausencia de precipitados en la zona afectada técnicamente, lo que vendría a confirmar la hipótesis de que estos son compuestos metaestables que se disuelven en la matriz $\alpha$ cuando la temperatura supera cierto umbral.

Del análisis químico confirma que las partículas precipitadas son compuestos Al-Mn o Al-Mn-Mg, sin que se hayan detectado trazas de fase $\beta \mathrm{Mg}_{17} \mathrm{Al}_{12}$, este hecho estaría de acuerdo lo indicado por otros autores (Ben Hamu et al., 2009) cuando afirman que la fase no es estable a temperatura ambiente, la gran cantidad de este tipo de partículas dispersas se podría explicar desde el punto de vista de la solubilidad del Mn en el Mg, según Avedesiam y Baker (1999) la solubilidad sólida del manganeso en el $\mathrm{Mg}$ está limitada, y ésta se reduce con la adición de aluminio, no superando el $0,3 \%$ en peso, de acuerdo con los datos del fabricante del material base la aleación a AZ31B empleada en este estudio tiene una contenido de manganeso comprendido entre un mínimo del 0,3 y un máximo del 1\%, es decir, el contenido es superior al límite máximo para la solubilidad sólida de este elemento en la matriz, lo que explicaría la presencia de partículas precipitadas o segregadas en el material base de forma previa la soldadura tal y como se indica en publicaciones anteriores (Segarra et al., 2015), de igual forma, sería lógico pensar que el empleo de material de aporte AZ92, con mayor contenido en aluminio, va a modificar la composición química al menos en la zona del material base fundida durante el proceso de soldadura, el incremento de aluminio en el cordón por tanto producirá una disminución de la solubilidad y la segregación de partículas precipitadas de manganeso, dicha variación de la solubilidad también justificaría la ausencia de partículas precipitadas en la zona afectada térmicamente de las muestras soldadas (Fig. 7a) tanto en las que emplean como material de aporte la aleación AZ31 como las que emplean la aleación AZ92, esta hipótesis también respaldaría la validez del modelo termomecánico desarrollado, si se observa la Fig. 7b, se aprecia que para el rango de contenido de manganeso del aleación AZ31 entre 0,3-1\% la línea de sólidus que marca la variación de la solubilidad del manganeso en la matriz de magnesio se encuentra por debajo de los valores temperatura de pico de $550{ }^{\circ} \mathrm{C}$ de los ciclos térmicos que experimenta el material base, quedaría por tanto explicado tanto la modificación del tamaño de grano como la variación de precipitados en la zona afectada térmicamente y su relación con los ciclos térmicos inducidos por la soldadura.

Las caracterizaciones mecánicas realizadas con las líneas de microdureza no muestran una mejora significativa de las propiedades mecánicas de las muestras soldadas con materiales más nobles.

El hecho de que la microdureza de los cordones de soldadura sea prácticamente la misma, se podría explicar teniendo en consideración que la aleación AZ92 es endurecible por precipitación, por lo que es más que probable que el cordón se encuentre parcialmente solubilizado y que la dispersión de precipitados no se adecuada declara la resistencia mecánica

En línea con lo indicado al tamaño de grano las muestras soldadas con material de aporte AZ92 al tener un tamaño de grano mayor en la ZAT que las soldadas con AZ31 presentan una disminución de sus propiedades mecánicas en esta zona, esto indica que a priori desde un punto de vista mecánico no parece que el empleo de una aleación con mayor contenido en aluminio y zinc, tal y como recomiendan Avedesiam y Baker (1999) represente una mejora significativa de las propiedades mecánicas de la unión soldada,

De acuerdo a los párrafos anteriores sería interesante investigar por un lado cuáles son los parámetros óptimos de la soldadura de este tipo de aleaciones de cara a minimizar la zona afectada térmicamente y comprobar si a través de un tratamiento térmico postsoldadura es posible recuperar la dispersión de partículas de manganeso sin que el incremento del tamaño de grano perjudique demasiado la resistencia mecánica de la unión, de esta manera también se podría evaluar que factor es más importante desde el punto de vista de la corrosión, bien el tamaño de grano o la proporción de partículas dispersas en la matriz y se podría comprobar si es posible mejorar las propiedades mecánicas de este tipo de uniones, comprobando para el caso de las muestras soldadas con material de aporte AZ92 si los valores de microdureza tan similares a los de las muestras soldadas con aleación AZ31 se puede mejorar con un tratamiento de endurecimiento por precipitación. 


\section{CONCLUSIONES}

- Se ha caracterizado la microestructura de la aleación AZ31B cuando es soldada, identificando las diferentes fases y microconstituyentes mediante microscopía óptica y electrónica de barrido.

- Se ha desarrollado un modelo de simulación termomecánico que permite obtener una respuesta fiable de la soldadura de estas aleaciones, facilitando la obtención de información que desde el punto de vista experimental sería muy complicado de determinar.

- Se ha obtenido gracias al modelo de simulación desarrollado las dimensiones de la zona afectada térmicamente y el ciclo térmico que produce la modificación de la microestructura del material base.

- Desde el punto de vista del diseño y la durabilidad, se ha justificado porque el empleo de materiales de aporte más nobles (mayor contenido de Aluminio y zinc) implica un empeoramiento de la resistencia a la corrosión de las uniones soldadas sin que apenas se observe una apreciable mejora de las propiedades mecánicas.

\section{AGRADECIMIENTOS}

Los autores quieren agradecer el soporte financiero del Departamento de Física Aplicada e Ingeniería de Materiales de la Escuela Superior de Ingenieros Industriales de Madrid de la Universidad Politécnica de Madrid.

\section{REFERENCIAS}

Avedesiam M., Baker H. (1999). ASM Speciality Handbook. Magnesium and Magnesium alloys. Ed. ASM International, Materials Park Ohio, USA, pp. 3-4.

Ben Hamu, G., Eliezer, D., Wagner, L. (2009). The relation between severe plastic deformation microstructure and corrosion behavior of AZ31 magnesium alloy. J. Alloy. Compd. 468 (1-2), 222-229. https://doi.org/10.1016/j.jallcom.2008.01.084.

Cheng, Y., Qin, T., Wang, H., Zhang, Z. (2009). Comparison of corrosion behaviors of AZ31, AZ91, AM60 and ZK60 magnesium alloys. T. Nonferr. Metal. Soc. China 19 (3), 517-524. https://doi.org/10.1016/S1003-6326(08)60305-2.

Dobrzanski, L.A., Tanski. T., Cizek, L., Brytan, Z. (2007). Structure and properties of magnesium cast alloys. J. Mater. Process. Tech. 192-193, 567-574. https://doi.org/10.1016/j. jmatprotec.2007.04.045.

Feliu, Jr. S., Maffiotte, C., Galván, J.C., Barranco, V. (2011). Atmospheric corrosion of magnesium alloys AZ31 and AZ61 under continuous condensation conditions. Corros. Sci. 53 (5), 1865-1872. https://doi.org/10.1016/j.corsci. 2011.02.003.

Kramer, D.A. (2001). Magnesium, its Alloys and Compounds. U.S. Geological Survey. Open-File Report 01-341. Disponible: https://pubs.usgs.gov/of/2001/of01-341/of01-341.pdf.

Merino, M.C., Pardo, A., Arrabal, R., Merino, S., Casajús, P., Mohedano, M. (2010). Influence of chloride ion concentration and temperature on the corrosion of $\mathrm{Mg}-\mathrm{Al}$ alloys in salt fog. Corros. Sci. 52 (5), 1696-1704. https://doi.org/ 10.1016/j.corsci.2010.01.020.

Pardo, A., Merino, M.C., Coy, A.E., Arrabal, R., Viejo, F., Matykina, E. (2008). Corrosion behaviour of magnesium/ aluminium alloys in 3.5 wt.\% NaCl. Corros. Sci. 50 (3), 823-834. https://doi.org/10.1016/j.corsci.2007.11.005.

Polmear, I.J. (2006). Light Alloys. $4^{\text {th }}$ Edition, Elsevier, England.

Segarra, J.A., Calderón, B., Portolés, A. (2015). Study of the corrosion behavior of magnesium alloy weldings in $\mathrm{NaCl}$ solutions by gravimetric tests. Rev. Metal. 51 (3), e050. https://doi.org/10.3989/revmetalm.050.

Song, G.-L., Xu, Z. (2012). Effect of microstructure evolution on corrosion of different crystal surfaces of AZ31 Mg alloy in a chloride containing solution. Corros. Sci. 54, 97-105. https://doi.org/10.1016/j.corsci.2011.09.005.

Te-Chang, T., Chih-Chung, Ch., Deng-Maw, T., Ko-Ta, Ch. (2011). Modeling and analyzing the effects of heat treatment on the characteristics of magnesium alloy joint welded by the tungsten-arc inert gas welding. Mater. Design 32 (8-9), 4187-4194. https://doi.org/10.1016/j.matdes.2011.04.040.

UNE-EN ISO 643 (2013). Aceros. Determinación micrográfica del tamaño de grano aparente. AENOR, Madrid.

Walton, C.A., Martin, H.J., Horstemeyer, M.F., Wang, P.T. (2012). Quantification of corrosion mechanisms under immersion and salt-spray environments on an extruded AZ31 magnesium alloy. Corros. Sci. 56, 194-208. https:// doi.org/10.1016/j.corsci.2011.12.008.

Zeng, R.-Ch., Zhang, J., Huang, W.-J., Dietzel, W., Kainer, K.U., Blawert, C., Ke, W. (2006). Review of studies on corrosion of magnesium alloys. T. Nonferr. Metal. Soc. China 16 (Suppl 2), s763-s771. https://doi.org/10.1016/ S1003-6326(06)60297-5. 